three died. ${ }^{1}$ With such a small relapse rate intensive radiological follow up is unnecessary, and four or five chest radiographs within the first two years after treatment are probably ample.

Although we strongly agree that close surveillance is essential in stage 1 teratoma, we question whether computed tomography beyond the initial investigation is required. Although about half of the $15-25 \%$ of patients who relapse have para-aortic disease, nearly all of these patients will be detected by virtue of raised tumour markers or associated lung metastases. Repeat abdominal computed tomography is therefore of value only for the few patients who relapse with disease in the abdomen without raised markers. There were no deaths in 45 patients followed up without repeat computed tomography, ${ }^{2}$ and this remains true of the current total of 93 .

These considerations would not, however, apply if only surveillance were to be considered in stage 1 seminoma since the para-aortic nodes are almost the only site at which relapse is likely to occur and there is currently no adequate marker available for seminoma. These facts together with the expense and the longer clinical course of seminoma support the benefits of a single radiotherapy course with minimal morbidity, after which further follow up is almost unnecessary.

GRAHAM READ R J JOHNSON B EDDLESTON

Christie Hospital and Holt Radium Institute

1 Read G, Robertson AG, Blair V. Radiotherapy in seminoma of the testis. Clin Radiol 1983;34:469-73 Read G, Johnson RJ, Wilkinson PM, Eddleston BE Prospective study of follow up only in stage 1
teratoma of the testis. $\mathrm{Br} \mathrm{Med} \mathcal{F} 1983 ; 287: 1503-5$.

\section{Trimethoprim resistance in Gram negative urinary pathogens}

SIR,-In 1983 I reported a sudden rise in 1982 of trimethoprim resistance in normally susceptible Gram negative urinary pathogens isolated in this laboratory. ${ }^{1}$ The rise was sharpest in organisms isolated from general practice patients, and I suggested that this might be due to the increasing use of trimethoprim alone outside hospital. In the two years since then we have persisted in our policy of not reporting the sensitivity of urinary organisms to trimethoprim alone, and I have analysed figures obtained in the same way for October 1983 and 1984 (see table). There has been a gradual decline in resistance, both in hospital and general practice isolates, the overall percentage having now returned to the level of 1979-81.

The original report received considerable publicity locally, and it is tempting to wonder whether the satisfactory improvement might be due to a change in prescribing habits. The ratio of the use of co-trimoxazole to trimethoprim in this hospital in 1982 was $16: 1$; in 1984 it was $19: 1$. When I wrote my previous report I had not been able to obtain

Percentage of normally susceptible Gram negative urinary pathogens resistant to trimethoprim. Figures in parentheses are number of isolates tested

\begin{tabular}{|c|c|c|c|c|c|c|c|}
\hline & 1978 & 1979 & 1980 & 1981 & 1982 & 1983 & 1984 \\
\hline $\begin{array}{l}\text { Hospital (inpatient and } \\
\text { outpatient) } \\
\text { General practice }\end{array}$ & $\begin{array}{l}\mathrm{NA} \\
\mathrm{NA}\end{array}$ & $\begin{array}{l}\text { NA } \\
\text { NA }\end{array}$ & $\begin{array}{l}\text { NA } \\
\text { NA }\end{array}$ & $\begin{array}{r}19(326) \\
7(521)\end{array}$ & $\begin{array}{l}24(381) \\
15(507)\end{array}$ & $\begin{array}{l}24(419) \\
12(701)\end{array}$ & $\begin{array}{l}16(382) \\
11(807)\end{array}$ \\
\hline Total & $9(709)$ & $12(821)$ & $13(794)$ & $12(847)$ & $19(888)$ & $17(1120)$ & 12 (1189) \\
\hline
\end{tabular}

NA $=$ Not available

figures for comparative use in general practice, but I have now compared the figures for the use of proprietary preparations for the year December 1981 to November 1982 with those for the year September 1983 to August 1984. In those areas served exclusively by this laboratory the ratio of the use of co-trimoxazole to trimethoprim was $17: 1$ in 1981-2 and 18:1 in 1983-4. These figures compare with $8: 1(1981-2)$ and $3: 1(1983-4)$ in an area served only by a laboratory which reports trimethoprim sensitivity of urinary pathogens, and $11: 1$ and $6: 1$ respectively in two areas which use our laboratory for some of their work. All the 1983-4 figures for co-trimoxazole are likely to be an underestimate because they refer only to proprietary preparations; over the past year there has been increasing use of generic co-trimoxazole and our laboratory figures suggest that generic prescribing

Clearly our laboratory policy has kept the level of trimethoprim prescribing well below that of other areas, and trimethoprim's use in comparison with that of co-trimoxazole has declined over the past two years, in marked contrast to the pattern in areas that have adopted a different policy. The overall level of trimethoprim resistance here has returned to that of $1979-81$

Public Health Laboratory,

St Mary's General Hospital,
Portsmouth, Hants PO3 6AQ

RoSALIND MASKELI

Maskell R. Trimethoprim resistance in Gram negative

\section{Calcium supplementation and} postmenopausal bone loss

SIR,-The paper by Dr Nilas and her colleagues (27 October, p 1103) contains data on which it would be helpful to have the authors' further comments, particularly if they have additional data.

(1) Three groups of normal women in the early menopause with low, medium, and high intakes of dietary calcium were given an additional $500 \mathrm{mg}$ of calcium daily. In each group the urinary calcium concentration at first rose over three to six months and then gradually returned to the basal value, or even below in the high intake group. What is happening here? Does the absorption of calcium at first increase and then gradually diminish, and, if so, might this not be important in the management and perhaps the aetiology of postmenopausal demineralisation?

(2) The authors state that all three groups showed a similar fall in bone mineral content over two years. Fig 2 shows quite clearly, however, that in the low intake group the fall occurred over the first 15 months and was then arrested. A similar effect may possibly have occurred in the high intake group after 21 months. Can the authors explain the arrest, perhaps from subsequent follow up of the low intake group?

(3) The medium intake subjects appear to fall into two clusters with respect to loss of 